\title{
More hospitals Choosing Wisely
}

A campaign that challenges doctors and patients to rethink "more-is-better" medicine is gaining traction in health organizations across Canada. Following in the footsteps of a popular American campaign, Choosing Wisely Canada works with medical societies to identify tests and treatments that are unnecessary and may even cause harm.

North American health care culture tends to err on the side of doing too much, says Dr. Wendy Levinson, chair of the campaign. A 2014 JAMA Internal Medicine study of American Medicare patients revealed that $25 \%-42 \%$ received at least one unnecessary test or treatment over the course of a year. A June 2 CMAJ study also showed frequent testing before low-risk surgical procedures, despite recommendations to limit this practice.

Levinson cites force of habit, fear of litigation and patient expectations among the factors driving overuse.

Choosing Wisely Canada is about creating a culture of questioning, she says, "and it's important for physicians to lead because they have a lot of influence over how health resources are spent." The Canadian Medical Association is a founding partner.

Since the April 2014 launch, more than 40 medical societies, representing the majority of Canadian physicians, have joined the campaign and 29 have published lists of procedures that doctors and patients should question.
Health organizations have also started projects based on the recommendations and meet monthly via webinar to share progress.

\section{Tech-savvy solutions in Toronto}

In September 2014, North York General Hospital in Toronto began embedding Choosing Wisely Canada recommendations in the clinical order sets and medical directives used to order lab tests.

"We've since seen a $40 \%$ reduction in laboratory testing from the emergency department on an ongoing basis, without any observable change in outcomes," says Mark Fam, program director of strategy and diagnostics at the hospital.

A February 2015 overhaul of the preoperative clinic has achieved similar reductions in testing and other kinds of preoperative care, Fam adds.

The project was relatively easy to implement because the hospital already had an electronic ordering system for tests. "We didn't have to create a whole new process to get these ideas discussed; we just went through our annual order set update."

\section{Mapping misuse in Vancouver}

Vancouver Coastal Health and Providence Health Care in British Columbia are tracking unnecessary medical imaging for uncomplicated low-back pain, simple adnexal cysts, uncomplicated headache, minor head trauma and advanced osteoarthritis.

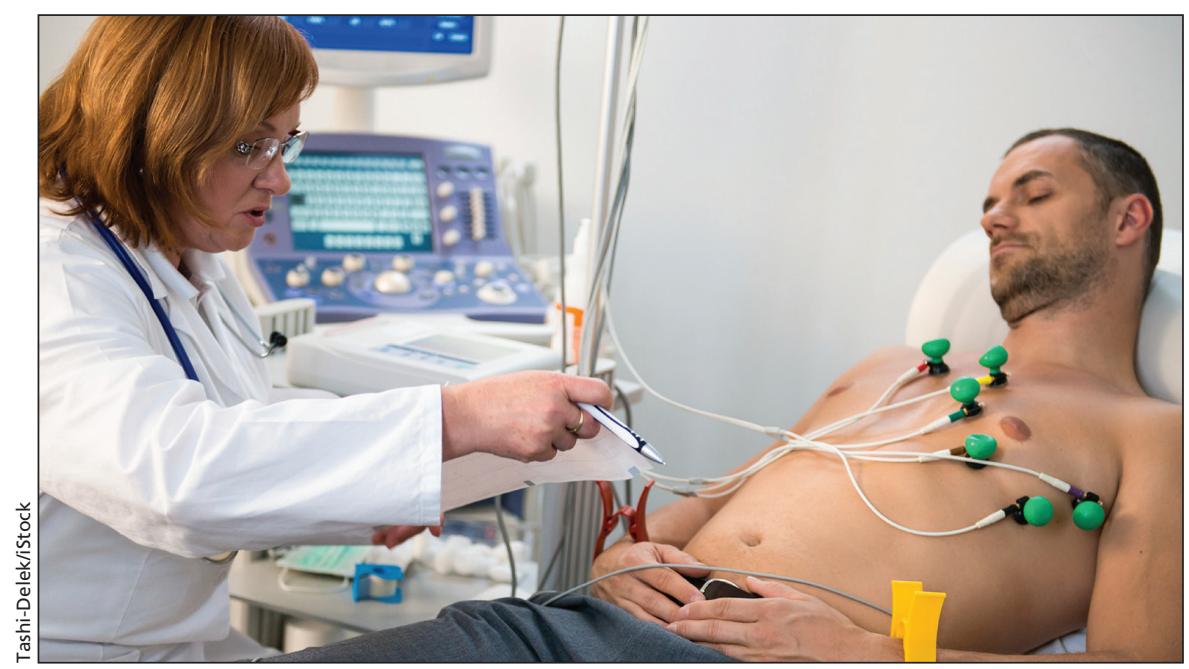

Is that test really necessary? One year into the Choosing Wisely Canada campaign, health organizations share their progress.
Dr. Bruce Forster, head of medical imaging for Vancouver Coastal Health, estimates $10 \%$ of tests may be inappropriate. An early look at the baseline data also suggests "significant variation" in how doctors are ordering tests.

The health authority is piloting a decision support tool that will prompt doctors to think twice before ordering certain exams.

\section{Spreading the word in Hamilton}

Other organizations have focused on education campaigns. St. Joseph's Healthcare Hamilton launched a sixmonth multimedia blitz in January 2015. Each month, the campaign promotes a different Choosing Wisely Canada recommendation, says Dr. Tamar Packer, medical director and head of newborn care. "There are paper signs, computer screen savers, email blasts and it's on the TV screens in the waiting rooms."

St. Joseph's also ran an education session on rational use of diagnostic imaging for local primary care doctors. A large percentage of the demand for imaging comes from doctors outside the hospital, and they're difficult to reach with quality improvement initiatives, says Packer. "It's a less cohesive group, with no organizational structure and no consequences if things don't change."

\section{Making the grade at $\mathrm{U}$ of $\mathrm{T}$}

Some medical students at the University of Toronto have asked their professors to include the Choosing Wisely Canada recommendations in lectures.

One of these students, Marisa LeonCarlyle, co-created a module on stewardship based on Choosing Wisely Canada principles for the Institute for Healthcare Improvement. The module breaks down the difference between cost and value, uses real-world examples of value in care and concludes with steps students can take to counter overuse. More than 4500 people have started the training.

"When you're teaching students to make medical decisions, it makes sense to incorporate value into that from day one," says Leon-Carlyle. — Lauren Vogel, CMAJ

CMAJ 2015. DOI:10.1503/cmaj.109-5078 